\title{
Comparación de los costos reales anuales asociados al manejo ambulatorio de pacientes hipertensos del programa cardiovascular en el Hospital Comunitario de Bulnes con el pago de prestaciones de Fonasa.
}

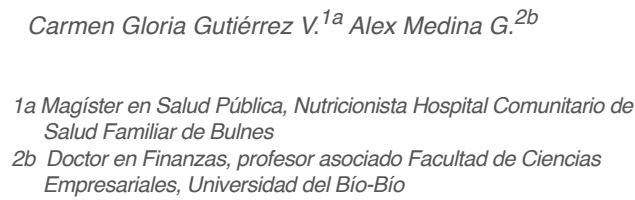

\section{Resumen:}

Antecedentes: El financiamiento de las prestaciones entregadas a los usuarios de atención primaria portadores de Hipertensión Arterial, esta determinado por un arancel definido por el Fondo Nacional de Salud (FONASA), el cual ha estimado montos a pagar a la institución prestadora, basados en estudios realizados a nivel nacional, los cuales difieren de las estimaciones de gasto real de cada prestación, para lo cual se hace necesario objetivar dicha diferencia de estimación a nivel local.

Objetivo: Determinar los costos directos e indirectos reales de las prestaciones entregadas a los usuarios hipertensos de control ambulatorio en atención primaria de salud mediante la metodología de costos asociados a actividades $(\mathrm{ABC})$, para compararlos con los valores asignados por FONASA.

Diseño: Estudio Analítico no experimental de enfoque cuantitativo de corte transversal, en que se revisaron
290 tarjetas de control de usuarios hipertensos bajo control en el Hospital Comunitario de Salud Familiar de Bulnes. Se analizaron específicamente los valores del control Médico, control Enfermera, control Nutricionista, y valores de exámenes: hematocrito, perfil lipídico, glicemia, creatinina plasmática, orina completa, potasio plasmático, electrocardiograma, atención farmacéutica.

Resultados: Los costos reales de las prestaciones incluidas en la Guía Clínica GES para el manejo ambulatorio de la Hipertensión Arterial, obtenidos mediante el uso de la metodología $\mathrm{ABC}$ fueron significativamente mayores a los establecidos por el FONASA (promedio miles de pesos: Fonasa 21.370; Costo real 39.991,7 \pm $11.999,4 ; \mathrm{p}<0,001)$.

Conclusión: Estos resultados sugieren que existe una diferencia entre lo que FONASA cancela por estas prestaciones y el gasto real en que se incurre por su atención. Si estos valores se acercan, podría obtenerse un mejor financiamiento del programa.

\section{Correspondencia:}

Nut. Carmen Gloria Gutiérrez V

Hospital Comunitario de Salud Familiar de Bulnes

Balmaceda 431. Bulnes

Fono: 56-42-585939

Carmeng.gutierrez@redsalud.gov.cl 


\section{Cost of ambulatory care of hypertensive subjects in a community hospital (bulnes): comparison of activity associated costs to the national health fund (fonasa) estimated payments in the setting of a national cardiovascular care program}

Background: Payments for ambulatory care of hypertensive patients in the national cardiovascular program is defined by FONASA based on national studies. Considerable differences are observed upon confrontation with the real costs involved in a given place.

Aim: to determine direct and indirect costs of health care actions in the ambulatory care of hypertensive patients through an activity based cost (ABC) methodology and to compare these with the corresponding payments defined by FONASA

Method: In a cross sectional design, 290 records of hypertensive subjects under control at the Community Hospital in Bulnes were analyzed. Costs of doctor, nurse and nutritionist controls were determined along

\section{Introducción:}

El Programa de Salud Cardiovascular, tiene como objetivo principal el manejo de la globalidad de los factores de riesgo de eventos cardiovasculares que pueden coexistir en un individuo determinado en un momento definido, a fin de reducir la probabilidad de ocurrencia de dichos eventos. ${ }^{1}$ Este programa ha recibido el refuerzo de la Ley de Garantías Explicitas en Salud (GES), mediante la cual se ha incorporado a la Hipertensión Arterial como patología principal de riesgo cardiovascular y para la que se han establecido plazos de intervención, flujogramas de atención y se han asignado recursos, mediante la valoración de una canasta de prestaciones que cada usuario deberá recibir en forma anual y que es financiada por el Fondo Nacional de Salud de Chile. ${ }^{2}$

En este estudio se comparó el valor pagado por el Fondo Nacional de Salud, por las prestaciones GES definidas en la Guía Clínica para Hipertensión Arterial ${ }^{3}$, para valorar si es que, este valor cubre efectivamente el gasto incurrido por el establecimiento en la realización o entrega de las prestaciones de salud de dicha patología.

El objetivo de este estudio fue, determinar los costos directos e indirectos reales de las prestaciones entregadas a los usuarios Hipertensos de manejo ambulatorio en atención primaria de salud mediante la metodología de costos asociados a actividades (ABC), para compararlos con los valores asignados por FONASA. ${ }^{4}$ with those related to laboratory tests including hematocrit, lipid profile, glycemia, plasma creatinine, urine analysis, plasma $\mathrm{K}$ level and electrocardiogram. Cost of pharmacy was also included.

Results: a monthly mean of $\$ 39992 \pm 12000$ per hypertensive patient cared was determined through $\mathrm{ABC}$ based costs. This is considerable greater than de $\$ 21370$ determined by FONASA $(\mathrm{p}<0.001)$.

\section{Conclusion: Payments considered by FONASA} are insufficient to cover real costs involved in ambulatory care of hypertensive patients. A better financing of the cardiovascular care program requires these costs and payments to converge.

Keywords: activity based costs, hypertension, cardiovascular care program.

\section{Material y Métodos:}

Estudio Analítico no experimental de enfoque cuantitativo y corte transversal, en que el universo estuvo compuesto por los Pacientes Hipertensos puros bajo control, en el Programa de Salud Cardiovascular del Hospital de Bulnes, correspondientes a 1.266 personas de la población asignada al establecimiento para el año 2010, distribuidas por sexo y grupo etáreo.

\section{Selección de la muestra:}

La muestra se seleccionó en base a la información entregada por el censo estadístico semestral del establecimiento, en el cual se encuentra la población bajo control de usuarios hipertensos, diferenciada por edad y sexo, mediante un muestreo estratificado proporcional, por edad, con un nivel de confianza de $95 \%$ y una diferencia esperada estimada, calculada en base a la variable índice costo total, respecto del valor que se obtendría, si se trabajara con el universo correspondido, para este estudio, de $\$ 1.000$, con lo que se obtuvo un tamaño muestral de 290 personas. Para determinar el tamaño de la muestra se utilizó una muestra piloto de 30 pacientes, con el fin de calcular la varianza.

Se seleccionaron las tarjetas de control, correspondientes a los sujetos Hipertensos, bajo control en el Programa de Salud Cardiovascular, previa estratificación proporcional del tamaño muestral siguiendo las proporciones del uni- 
verso, mediante la técnica de azar simple, para a continuación realizar un tamizaje que permita determinar si cada unidad de análisis cumple con los criterios de inclusión establecidos, efectuando reposición de los casos que no cumplían satisfactoriamente con ellos.

Criterios de Inclusión: Usuarios Hipertensos primarios puros, en control en el Programa de Salud Cardiovascular, durante el año 2010, mayores de 20 años, diagnosticados de acuerdo a los protocolos establecidos, asistentes regulares a las intervenciones definidas para el manejo ambulatorio de la patología, en el nivel primario de atención, diagnosticados con anterioridad al mes de marzo 2010.

Seleccionadas las tarjetas de control, se procedió a extraer de ellas la información correspondiente a consultas, exámenes de laboratorio y tratamiento farmacológico, registradas en el período de estudio, completando la información mediante la revisión de la ficha clínica del usuario.

\section{Categorización de usuarios:}

Los usuarios Hipertensos bajo control en el establecimiento, fueron categorizados en 3 etapas, de acuerdo a las cifras de presión arterial presentadas, de acuerdo a los parámetros establecidos en la Guía Clínica GES para Hipertensión Arterial Primaria en mayores de 15 años. ${ }^{3}$

Costos Directos: Se consideraron como tales, el recurso humano involucrado, materiales e insumos.

1.- Recurso Humano de intervención directa: $\mathrm{Se}$ categorizó el Recurso Humano de acuerdo a su estamento, definiendo el tiempo destinado a cada proceso de cada uno de ellos. Considerando el promedio de los Ingresos totales (total haberes) por estamento, se calculó el número de días efectivamente trabajados, obtenido de la diferencia entre los días hábiles y la suma de días feriado, administrativos y de capacitación de cada estamento, a partir de lo cual se calculó el valor hora, con lo cual se obtuvo el valor del tiempo invertido en cada prestación por unidad de tiempo.

2.- Equipos, instrumentos y muebles de uso directo: Corresponden a todos los elementos que estaban disponibles en el recinto donde se realizó la intervención. Fueron valorados a costo de reposición, para lo cual se utilizaron los precios de mercado en el momento que se costeó la intervención. Estos precios se aplicaron al período de vida útil del bien, dividiéndolos por la fracción de tiempo invertido en la entrega de la prestación.

3.- Insumos: Corresponde a los insumos directos utilizados en la entrega del servicio. Entre ellos destacan: fármacos de tratamiento hipertensión arterial, jeringa y tubo recolector de muestra de sangre, frasco para recolectar muestra orina, cuyos valores unitarios se multiplicaron por el número de dosis de fármacos por usuario en el pe- ríodo o por el número de extracciones de muestras de sangre o recolecciones de orina, respectivamente.

Costos Indirectos: Se consideraron como tales la depreciación de la Infraestructura, bienes muebles, maquinarias, y los costos de Administración, así como de las unidades que apoyan al servicio: farmacia, aseo y personal. Estos costos se distribuyeron, por medio del método de Costeo Basado en Actividades $(\mathrm{ABC})^{5}$ de acuerdo al siguiente criterio.

1.- Determinación de unidades de apoyo clínico y administrativo que prestaron servicios de apoyo a las prestaciones directas, y entre las que se contaban Farmacia y Laboratorio, Sección orientación médico-estadístico (SOME), servicios generales, abastecimiento, contabilidad y recurso humano.

2.- Depreciación Infraestructura: En el estudio sólo era necesario conocer el costo de la depreciación asociado al uso de la infraestructura, ya que el costo de uso de equipamiento se calculó directamente.

Suponiendo una relación directa entre bienes de capital y gasto operacional, fue posible estimar la depreciación de la infraestructura, en un 5\% del gasto operacional total, el cual comprende: el gasto en recurso humano (subtitulo 21 del presupuesto) y el gasto en bienes y servicios de consumo, (subtitulo 22 del presupuesto). ${ }^{6}$

Determinación costos atención de Hipertensos: La determinación de costo real de los controles de Médico, Enfermera y Nutricionista, así como el de atención farmacéutica se realizó con el cálculo de tiempo ocupado en la realización de la actividad, el cual se utilizó, además, para la determinación de los costos de los demás ítems de intervención directa e indirecta, tal como se muestra en las tablas 3 y 4.

\section{Comparación gasto real prestaciones y valores Fona-} sa:

Los valores pagados por FONASA para las distintas prestaciones, se extrajeron de la Planilla de Pago de Prestaciones en Modalidad de Atención Institucional ${ }^{4}$, mientras que el gasto real se obtuvo del análisis estadístico de la base de datos, la que incluía la cantidad de prestaciones recibidas por cada usuario de la muestra y su comparación se detalla en la tabla 5 .

\section{Tabulación de la Información}

La información fue traspasada a una planilla Excel, en la que se incluyo para cada unidad de análisis, las prestaciones recibidas en cantidad y costo real determinado para cada una de ellas, la cual se analizó utilizando prueba de diferencia de medias t de student, entre el costo real de las prestaciones totales entregadas en el período en estudio y 
el costo definido por FONASA para dichas prestaciones, mediante el programa estadístico STATA versión 9.0

\section{Resultados:}

\begin{tabular}{|c|c|c|c|c|c|c|c|c|}
\hline \multicolumn{9}{|c|}{ ETAPAS } \\
\hline \multirow[t]{2}{*}{ EDAD } & \multicolumn{2}{|l|}{1} & \multicolumn{2}{|l|}{2} & \multicolumn{2}{|c|}{3} & \multicolumn{2}{|c|}{ TOTAL } \\
\hline & n & $\%$ & $n$ & $\%$ & n & $\%$ & $n$ & $\%$ \\
\hline $20-44$ años & 19 & 7,9 & 0 & 0 & 5 & 29,4 & 24 & 8,3 \\
\hline $45-64$ años & 96 & 39,8 & 22 & 68,8 & 8 & 47,1 & 126 & 43,4 \\
\hline 65 y mas años & 126 & 52,3 & 10 & 31,2 & 4 & 23,5 & 140 & 48,3 \\
\hline TOTAL & 241 & 100 & 32 & 100 & 17 & 100 & 290 & 100 \\
\hline Porcentaje & $83,1 \%$ & & $0 \%$ & & $5,9 \%$ & & & \\
\hline
\end{tabular}

En la tabla 1 se observa que el mayor porcentaje de individuos se encuentra en el grupo etáreo de 65 y más años $(\mathrm{n}=140)$, seguido del grupo de 45 a 64 años $(\mathrm{n}=126)$, mientras que el grupo menor lo constituyen los individuos de 20 a 44 años $(n=24)$.

\begin{tabular}{|c|c|c|c|c|c|c|c|c|}
\hline \multicolumn{9}{|c|}{ ETAPAS } \\
\hline \multirow[t]{2}{*}{ SEXO } & \multicolumn{2}{|l|}{1} & \multicolumn{2}{|l|}{2} & \multicolumn{2}{|c|}{3} & \multicolumn{2}{|c|}{ TOTAL } \\
\hline & $\mathbf{n}$ & $\%$ & $\mathrm{n}$ & $\%$ & $\mathrm{n}$ & $\%$ & $\mathrm{n}$ & $\%$ \\
\hline Femenino & 147 & 61 & 20 & 62,5 & 10 & 58,8 & 177 & 61,03 \\
\hline Masculino & 94 & 39 & 12 & 37,5 & 7 & 41,2 & 113 & 38,97 \\
\hline TOTAL & 241 & 100 & 32 & 100 & 17 & 100 & 290 & 100 \\
\hline
\end{tabular}

$\chi^{2}=0,064(2 g l), p=0.969$

En la tabla 2 se observa que, si bien existe una diferencia significativa entre la proporción de individuos de género femenino respecto del género masculino, esta distribución no presenta diferencias estadísticamente significativas en las tres etapas de categorización de Hipertensión Arterial.

\begin{tabular}{|c|c|c|c|c|}
\hline ITEM & $\begin{array}{l}\text { Médico } \\
\text { (12 min) }\end{array}$ & $\begin{array}{l}\text { Enfermera } \\
(15 \mathrm{~min})\end{array}$ & $\begin{array}{c}\text { Nutricionista } \\
\text { (15 min) }\end{array}$ & $\begin{array}{c}\text { Atención } \\
\text { Farmacéutica } \\
\text { ( } 7 \text { min) }\end{array}$ \\
\hline Recurso Humano & 3508,4 & 3311,6 & 3175,6 & 1789,2 \\
\hline Equipamiento & 3,02 & 4,98 & 4,87 & 5,4 \\
\hline Insumos & 8,1 & 13,4 & 13,4 & 7,43 \\
\hline Consumos básicos & 90 & 150 & 150 & 52,5 \\
\hline Infraestructura & 338,4 & 564 & 564 & 94,3 \\
\hline TOTAL & 3947,7 & 4043,6 & 3907,5 & 1948,8 \\
\hline
\end{tabular}

\begin{tabular}{|c|c|c|c|c|c|c|c|}
\hline ITEM & $\begin{array}{l}\text { ematocrito } \\
\text { (15 min) }\end{array}$ & $\begin{array}{c}\text { Creatinina } \\
\text { Plasmática } \\
\text { ( } 7 \text { min) }\end{array}$ & $\begin{array}{l}\text { Orina } \\
\text { Completa } \\
(30 \mathrm{~min})\end{array}$ & $\begin{array}{l}\text { Glicemia } \\
\text { (7 min) }\end{array}$ & $\begin{array}{l}\text { Perfil } \\
\text { lipídico } \\
\text { (7 min) }\end{array}$ & $\begin{array}{l}\text { Potasio } \\
\text { Plasmátic (1) } \\
\text { (16 min) }\end{array}$ & $\begin{array}{l}\text { ECG } \\
15 \mathrm{~min} \text { ) }\end{array}$ \\
\hline RRHH & 2308 & 941,3 & 5304,5 & 941,3 & 941,3 & 2479 & 1313,7 \\
\hline Equipamiento & 7,5 & 3,5 & 15,1 & 3,5 & 3,5 & 8 & 10,2 \\
\hline Insumos & 948,1 & 322,7 & 471,1 & 177,7 & 536,7 & 318,7 & 70,7 \\
\hline C. básicos & 112,5 & 52,5 & 225 & 52,5 & 52,5 & 120 & 112,5 \\
\hline Infraestructura & 423 & 197,4 & 846 & 197,4 & 197,4 & 451,2 & 423 \\
\hline TOTAL & 3800 & 1519 & 6868 & 1374 & 1733 & 3381 & 1930 \\
\hline
\end{tabular}

En las tablas 3 y 4 se concentran los costos reales directos e indirectos de las distintas prestaciones entregadas a los usuarios hipertensos bajo control y sus respectivos totales, en los cuales es posible observar que el recurso humano es el ítem que ocupa la mayor proporción del total del costo real, con porcentajes que varían desde 57,3\% en el examen de perfil lipídico hasta el 91,8\% en la atención farmacéutica.

\begin{tabular}{|c|c|c|c|}
\hline \multicolumn{4}{|c|}{ costo (\$) } \\
\hline PRESTACIÓN & FONASA & REAL & $\mathbf{P}$ \\
\hline Control Médico & 3710 & $4152,2 \pm 2324,9$ & $<0,001$ \\
\hline Control Enfermera & 990 & $4952,2 \pm 2691,9$ & $<0,001$ \\
\hline Control Nutricionista & 990 & $3234,2 \pm 2614,8$ & $<0,001$ \\
\hline Hematocrito & 450 & $3773,8 \pm 1611,7$ & $<0,001$ \\
\hline Perfil Lipídico & 4110 & $1721,0 \pm 735,0$ & 1,0000 \\
\hline Glicemia & 870 & $1364,5 \pm 582,7$ & $<0,001$ \\
\hline Creatinina Plasmática & 870 & $1503,3 \pm 650,3$ & $<0,001$ \\
\hline Orina Completa & 1240 & $6820,6 \pm 2912,9$ & $<0,001$ \\
\hline Potasio Plasmático & 970 & $3322,7 \pm 1419,1$ & 0,001 \\
\hline Electrocardiograma & 4340 & $785,3 \pm 949,8$ & 1,000 \\
\hline Atención Farmacéutica & 1780 & $8321,7 \pm 5179,44$ & $<0,001$ \\
\hline TOTAL & 21370 & $39991,7 \pm 11999,4$ & $<0,001$ \\
\hline
\end{tabular}

Prueba de $t$ student

En la tabla 5, se resumen los costos promedio reales de cada prestación entregada, observándose que estos son significativamente menores $(\mathrm{p}<0,001)$ al valor pagado por FONASA, a excepción del examen perfil lipídico y electrocardiograma.

\section{Discusión:}

El análisis de los resultados obtenidos en este estudio, muestra que la media del gasto real total de los usuarios hipertensos bajo control es mayor que el valor pagado por 
FONASA por las prestaciones establecidas en la canasta GES. Este estudio comparó los costos anuales reales asociados al tratamiento ambulatorio de la Hipertensión Arterial Primaria de acuerdo al conjunto de prestaciones establecidas en la Guía Clínica Ges 2005, actualizada para el año 2009, encontrándose que los costos reales de las prestaciones entregadas tienen valores mayores a los establecidos por FONASA, y en los cuales destaca el factor recurso humano, como el de mayor relevancia dentro del costo total real de cada prestación, con porcentajes que varían desde un 57,3\% en el examen de perfil lipídico hasta un $91,8 \%$ en la atención farmacéutica, a diferencia de los factores de equipamiento e insumo, los cuales representan menos del $1 \%$ del total de dicho costo en cada una de las prestaciones estudiadas.

En el estudio de verificación de costo esperado por beneficiario ${ }^{2}$, del conjunto priorizado de problemas de salud con garantías explícitas realizado el 2007, se utilizó el sistema de costeo de intervenciones, que diferencia entre costos directos e indirectos y que requiere de la existencia de centros de costos para la asignación de costos indirectos a las prestaciones.

El estudio de costo efectividad de intervenciones en Salud Pública ${ }^{6}$, utiliza también la metodología de determinación de costos directos e indirectos, con el fin de relacionar costos de intervenciones con efectos de índole biológica (años de vida ganados, disminución de AVISA). Si bien no se dispone de estudios nacionales que caractericen los costos reales en que se incurre en el proceso de atención de usuarios portadores de patologías crónicas, en un estudio retrospectivo de costos de tratamientos intensivos por paciente y día cama ${ }^{7}$, se midió los costos directos e indirectos ocasionados con motivo de la hospitalización en unidades de cuidados intensivos del Hospital de $\mathrm{Cu}$ ricó, basados en el Sistema de Costos Basados en Actividades, encontrando que el mayor porcentaje de los costos asociados, corresponde al factor recurso humano, el cual fluctúa entre el $66 \%$ y $77 \%$, seguido del ítem exámenes de laboratorio con una fluctuación entre $10 \%$ y $15 \%$, para finalizar con el ítem de medicamentos con una representatividad del $10 \%$.

La principal limitación de este estudio, está dada por la inexistencia de centros de costos, lo que dificulta la obtención de costos indirectos para las distintas prestaciones identificadas, debiendo idearse métodos de determinación, que si bien permiten una aproximación, pueden tener errores de omisión de determinados ítems. Por otro lado, el sistema de registro de las atenciones recibidas por los usuarios bajo control, podría omitir algunas de ellas, contribuyendo a subestimar el gasto real total. Por otro lado, conviene analizar la representatividad de estos datos en el contexto provincial, regional y nacional, toda vez que no se encuentran disponibles datos que permitan establecer dicha representatividad.

\section{Referencias:}

1. MINISTERIO DE SALUD. REORIENTACIÓN DE LOS PROGRAMAS DE HIPERTENSIÓN Y DIABETES. Programa de Salud Cardiovascular. Chile. 2002. [en línea]

http://www.redsalud.gov.cl/portal/url/page/minsalcl/g_ proteccion/g_cardiovascular/presentacion_programacardiovascular.html [consulta: 15 septiembre 2010]

2. MINISTERIO DE SALUD. Verificación del costo esperado por beneficiario del conjunto priorizado de problemas de salud con Garantías Explicitas. 2007: 61-90

[En línea] http://www.redsalud.gov.cl/portal/url/page/ minsalcl/g_temas/g_economia_dela salud/economiadelasalud.html [consulta: 08 octubre 2010]

3. MINISTERIO DE SALUD, GUÍA CLÍNICA HIPERTENSIÓN ARTERIAL PRIMARIA O ESENCIAL EN PERSONAS DE 15 AÑOS Y MÁS.1 ${ }^{\text {a }}$ edición. Santiago: Minsal 2009. [en línea]
$<$ http://www.redsalud.gov.cl/portal/url/page/minsalcl/g_gesauge/guias.html>

[consulta: 16 septiembre 2010]

4. FONDO NACIONAL DE SALUD. Planilla Modalidad Atención Institucional 2010. [en línea]

$<$ http://200.51.172.210/mai/aplic_mai.html> [consulta: 08 octubre 2010]

5. HORNGREN, CHARLES, FOSTER G, DATAR S. Introducción a los términos y propósitos de costo, en Contabilidad de Costos: un enfoque gerencial ( $12^{\circ}$ ed.). Prentice Hall Hispanoamericana S.A. México (2007). Pág 33.

6. CONCHA M, Y COL. Estudio de Costo Efectividad de Intervenciones para las Principales problemas de Salud Publica. Ministerio de Salud. Chile. 1999: 46 - 49.

7. ALVEAR S, CANTEROS J, RODRIGUEZ P. Estudio retrospectivo de costos de tratamientos intensivos por paciente y día cama. Rev. Médica de Chile 2010; 138:558-566. 